\title{
Existence Solution for 5th Order Differential Equations under Some Conditions
}

\author{
Sayada Nabhan Odda \\ Department of Mathematics, Faculty of Computer Science, \\ Qassim University, Burieda, Saudi Arabia \\ E-mail: nabhan100@yahoo.com \\ Received May 12, 2010; July 30, 2010; August 5, 2010
}

\begin{abstract}
We study a nonlinear differential equations in the Banach space of real functions and continuous on a bounded and closed interval. With the help of a suitable theorems (fixed point) and some boundary conditions, the $5^{\text {th }}$ order nonlinear differential equations has at least one positive solution.
\end{abstract}

Keywords: Fixed-Point Theorem, Banach Space, Nonlinear $5^{\text {th }}$ Order Differential Equations

\section{Introduction}

In this paper, we are going to study the solvability nonlinear $5^{\text {th }}$ order differential equations. We will look for solutions of that equation in the Banach space. The main tool used in our investigations to existence of positive solutions for the nonlinear 5th order boundary value problems. Let us mention that the theory of nonlinear differential equations has many useful applications in describing numerous events and problems of the real world. For example, nonlinear differential equations are often applicable in engineering, mathematical physics, economics and biology [1,2]. The nonlinear $5^{\text {th }}$ order differential equations studied in this paper is an existence and nonexistence of positive solutions by using object of mathematical investigations [3-5]. The results presented in this paper seem to be new and original. They generalize several results obtained up to now in the study of nonlinear differential equations of several types.

\section{Notation, Definition and Auxiliary Results}

\section{Theorem $2.1[6,7]$}

Assume that $U$ is a relatively open subset of convex set $K$ in Banach space $E$. Let $N: \bar{U} \rightarrow K$ be a compact map with $o \in U$. Then either

1) $N$ has a fixed point in $\bar{U}$; or

2) There $u \in U$ and $\lambda \in(0,1)$ such that $u=\lambda N u$.

Definition 2.1 An operator is called completely con- tinuous if it is continuous and maps bounded sets into pre-compacts.

Definition 2.2 Let $E$ be a real Banach space. A nonempty closed convex set $K \subset E$ is called cone of $E$ if it satisfies the following conditions:

1) $x \in K, \sigma \geq 0$ implies $\sigma x \in K$;

2) $x \in K,-x \in K$ implies $x=0$.

\section{Main Result}

In this section, we will study the existence and nonexistence of positive solutions for the nonlinear boundary value problem:

$$
\begin{gathered}
u^{(5)}(t)=f(t, u(t)), 0<\mathrm{t}<1, \\
u(0)=u^{\prime}(0)=u^{\prime \prime \prime}(0)=u^{(4)}(1)=0, \\
\alpha u^{\prime}(1)+\beta u^{\prime \prime}(1)=0, \\
\text { where } \beta, \alpha \geq 0, \quad \alpha+\beta>0
\end{gathered}
$$

Theorem 3.1.Under conditions (3.2) and (3.3), Equation (3.1) has a unique solution.

Proof: Applying the Laplace transform to Equation (3.1) we get,

$$
\begin{aligned}
& s^{5} \bar{u}(s)-s^{4} u(0)-s^{3} u^{\prime}(0)-s^{2} u^{\prime \prime}(0) \\
& -s u^{\prime \prime \prime}(0)-u^{4}(0)=\bar{y}(s)
\end{aligned}
$$

Where $\bar{u}(s)$ and $\bar{y}(\mathrm{~s})$ is the Laplace transform of $u(t)$ and $y(t)$ respectively. The Laplace inversion of Equation (3.4) gives the final solution as: 


$$
\begin{aligned}
& u(t)=\frac{\alpha+3 \beta}{[\alpha+\beta] 3 !} \int_{0}^{1} \frac{t^{2}}{2 !} f(s, u(s)) d s-\frac{\alpha}{[\alpha+\beta] 3 !} \int_{0}^{1} \frac{t^{2}(1-s)^{3}}{2 !} f(s, u(s)) d s \\
& -\frac{\beta}{[\alpha+\beta] 2 !} \int_{0}^{1} \frac{t^{2}(1-s)^{2}}{2 !} f(s, u(s)) d s-\int_{0}^{1} \frac{t^{4}}{4 !} f(s, u(s)) d s+\int_{0}^{t} \frac{(t-s)^{4}}{4 !} f(s, u(s)) d s
\end{aligned}
$$

The proof is complete.

Defining $T: X \rightarrow X$ as:

$$
\begin{aligned}
& T u(t)=\frac{\alpha+(3) \beta}{[\alpha+\beta](3) !} \int_{0}^{1} \frac{t^{2}}{2 !} f(s, u(s)) d s-\frac{\alpha}{[\alpha+\beta](3) !} \int_{0}^{1} \frac{t^{2}(1-s)^{3}}{2 !} f(s, u(s)) d s \\
& -\frac{\beta}{[\alpha+\beta](2) !} \int_{0}^{1} \frac{t^{2}(1-s)^{2}}{2 !} f(s, u(s)) d s-\int_{0}^{1} \frac{t^{4}}{(4) !} f(s, u(s)) d s+\int_{0}^{t} \frac{(t-s)^{4}}{(4) !} f(s, u(s)) d s
\end{aligned}
$$

Where $\mathrm{X}=\mathrm{C}[0,1]$ is the Banach space endowed with the supper norm. We have the following result for operator $\mathrm{T}$.

Lemma 3.1
Assume that $f:[0,1] \times R \rightarrow R$ is continuous function, then $\mathrm{T}$ is completely continuous operator.

Proof: It is easy to see that $\mathrm{T}$ is continuous. For $u \in M=\{u \in X ;\|u\| \leq l, l>0\}$, we obtain,

$$
\begin{aligned}
& T u(t)=\left|\begin{array}{l}
\frac{\alpha+3 \beta}{[\alpha+\beta] 3 !} \int_{0}^{1} \frac{t^{2}}{2 !} f(s, u(s)) d s-\frac{\alpha}{[\alpha+\beta] 3 !} \int_{0}^{1} \frac{t^{2}(1-s)^{3}}{2 !} f(s, u(s)) d s \\
-\frac{\beta}{[\alpha+\beta] 2 !} \int_{0}^{1} \frac{t^{2}(1-s)^{2}}{2 !} f(s, u(s)) d s-\int_{0}^{1} \frac{t^{4}}{4 !} f(s, u(s)) d s+\int_{0}^{t} \frac{(t-s)^{4}}{4 !} f(s, u(s)) d s
\end{array}\right| \\
& \leq \frac{\alpha+3 \beta}{[\alpha+\beta] 3 !} \int_{0}^{1} \frac{t^{2}}{2 !}|f(s, u(s))| d s+\frac{\alpha}{[\alpha+\beta] 3 !} \int_{0}^{1} \frac{t^{2}(1-s)^{3}}{2 !}|f(s, u(s))| d s \\
& +\frac{\beta}{[\alpha+\beta] 2 !} \int_{0}^{1} \frac{t^{2}(1-s)^{2}}{2 !}|f(s, u(s))| d s+\int_{0}^{1} \frac{t^{4}}{4 !}|f(s, u(s))| d s+\int_{0}^{t} \frac{(t-s)^{4}}{4 !}|f(s, u(s))| d s \\
& \leq \frac{\alpha+3 \beta}{[\alpha+\beta] 3 !} \frac{L}{2 !}+\frac{\alpha}{[\alpha+\beta] 4 !} \frac{L}{2 !}+\frac{\beta}{[\alpha+\beta] 3 !} \quad \frac{L}{2 !}+\frac{L}{4 !}+\frac{L}{5 !}
\end{aligned}
$$

where $L=\max _{0 \leq t \leq 1,|u| \mid \leq 1}|f(t, u(t))|+1$, tinuity of $\overline{T(M)} . \forall u \in M, \forall \varepsilon>0, t_{1}<t_{2} \in[0,1]$.

so $\mathrm{T}(\mathrm{M})$ is bounded. Next we shall show the equicon-

Let

$$
\begin{aligned}
& \eta<\operatorname{Min}\left\{\begin{array}{l}
2 ! \frac{\varepsilon[\alpha+\beta] 3 !}{5 L[\alpha+3 \beta]}, 2 ! \frac{\varepsilon[\alpha+\beta] 4 !}{5 L \alpha} \\
2 ! \frac{\varepsilon[\alpha+\beta] 3 !}{5 L \beta}
\end{array}\right\}, \sigma<\left\{\frac{\varepsilon(4) !}{5 L}\right\}, \gamma<\left\{\varepsilon \frac{5 !}{5 L}\right\}, \mathrm{t}^{2}{ }_{2}-\mathrm{t}^{2}{ }_{1}<\eta, \quad \mathrm{t}_{2}^{4}-\mathrm{t}_{1}^{4}<\sigma, \mathrm{t}_{2}^{5}+\mathrm{t}_{1}^{5}<\gamma \\
& \left|T u\left(t_{2}\right)-T u\left(t_{1}\right)\right|=\left|\begin{array}{l}
\frac{\alpha+3 \beta}{[\alpha+\beta] 3 !} \int_{0}^{1} \frac{t_{2}{ }^{2}-t_{1}^{2}}{2 !} f(s, u(s)) d s-\frac{\alpha}{[\alpha+\beta] 3 !} \int_{0}^{1} \frac{\left(t_{2}{ }^{2}-t_{1}^{2}\right)(1-s)^{3}}{2 !} f(s, u(s)) d s \\
-\frac{\beta}{[\alpha+\beta] 2 !} \int_{0}^{1}\left(\frac{\left.t_{2}{ }^{2}-t_{1}^{2}\right)(1-s)^{2}}{2 !} f(s, u(s)) d s-\int_{0}^{1} \frac{t_{2}{ }^{4}-t_{1}^{4}}{4 !} f(s, u(s)) d s\right. \\
+\int_{0}^{t_{2}} \frac{\left(t_{2}-s\right)^{4}}{4 !} f(s, u(s)) d s-\int_{0}^{t_{1}} \frac{\left(t_{1}-s\right)^{4}}{4 !} f(s, u(s)) d s
\end{array}\right| \\
& \leq \frac{\alpha+3 \beta}{[\alpha+\beta] 3 !} \frac{L\left(t_{2}^{2}-t_{1}^{2}\right)}{2 !}+\frac{\alpha}{[\alpha+\beta] 4 !} \frac{L\left(t_{2}^{2}-t_{1}^{2}\right)}{2 !}+\frac{\beta}{[\alpha+\beta](3) !} \frac{L\left(t_{2}^{2}-t_{1}^{2}\right)}{2 !}+\frac{L\left(t_{2}^{4}-t_{1}^{4}\right)}{4 !}+\frac{L t_{1}^{5}}{5 !}+\frac{L t_{1}^{5}}{5 !} \\
& \leq \frac{\alpha+3 \beta}{[\alpha+\beta] 3 !} \frac{L \eta}{2 !}+\frac{\alpha}{[\alpha+\beta] 4 !} \frac{L \eta}{2 !}+\frac{\beta}{[\alpha+\beta] 3 !} \quad \frac{L \eta}{2 !}+\frac{L \sigma}{4 !}+\frac{L \gamma}{5 !} \leq \frac{\varepsilon}{5}+\frac{\varepsilon}{5}+\frac{\varepsilon}{5}+\frac{\varepsilon}{5}+\frac{\varepsilon}{5} \text {. }
\end{aligned}
$$


Thus $\overline{T(M)}$ is equi-continuous. The Arzela-Ascoli theorem implies that the operator $\mathrm{T}$ is completely continuous.

\section{Theorem 3.2}

Assume that $f:[0,1] \times R \rightarrow R$ is continuous function, and there exist constants

$0<\mathrm{c}_{1}<\left(5 \frac{2 !(\alpha+\beta) 4 !}{n \alpha+(4)^{2} \beta}, \frac{5 !}{6}\right), \mathrm{c}_{2}>0$, such that

$|f(t, u(t))| \leq c_{1}|u|+c_{2}$ for all $t \in[0,1]$. Then the boundary value problem (3.1),(3.2)and(3.3) has a solution.
Proof: Following [8,9], we will apply the nonlinear alternative theorem to prove that $\mathrm{T}$ has one fixed point.

Let $\Omega=\{u \in X ;\|u\|<R\}$, be open subset of $X$, where

$$
R>\left\{4\left\{\begin{array}{c}
\frac{5 \alpha+(4)^{2} \beta}{2 !(4) !(\alpha+\beta)} c_{1}, \frac{6 c_{1}}{5 !}, \\
\frac{5 \alpha+(4)^{2} \beta}{2 !(4) !(\alpha+\beta)} c_{2}, \frac{6 c_{2}}{5 !}
\end{array}\right\}\right. \text {. }
$$

We suppose that there is a point $u \in \partial \Omega$ and $c_{1} \in(0,1)$ such that $u=T u$. So, for $u \in \partial \Omega$, we have:

$$
\begin{aligned}
& T u(t)=\left|\begin{array}{l}
\frac{\alpha+3 \beta}{[\alpha+\beta] 3 !} \int_{0}^{1} \frac{t^{2}}{2 !} f(s, u(s)) d s-\frac{\alpha}{[\alpha+\beta] 3 !} \int_{0}^{1} \frac{t^{2}(1-s)^{3}}{2 !} f(s, u(s)) d s \\
-\frac{\beta}{[\alpha+\beta] 2 !} \int_{0}^{1} \frac{t^{2}(1-s)^{2}}{2 !} f(s, u(s)) d s-\int_{0}^{1} \frac{t^{4}}{4 !} f(s, u(s)) d s+\int_{0}^{t} \frac{(t-s)^{4}}{4 !} f(s, u(s)) d s
\end{array}\right| \\
& \leq \frac{\alpha+3 \beta}{[\alpha+\beta] 3 !} \int_{0}^{1} \frac{t^{2}}{2 !}|f(s, u(s))| d s+\frac{\alpha}{[\alpha+\beta] 3 !} \int_{0}^{1} \frac{t^{2}(1-s)^{3}}{2 !}|f(s, u(s))| d s \\
& +\frac{\beta}{[\alpha+\beta] 2 !} \int_{0}^{1} \frac{t^{2}(1-s)^{2}}{2 !}|f(s, u(s))| d s+\int_{0}^{1} \frac{t^{4}}{4 !}|f(s, u(s))| d s+\int_{0}^{t} \frac{(t-s)^{4}}{4 !}|f(s, u(s))| d s
\end{aligned}
$$

So,

$$
\begin{aligned}
& T u(t)=\left|\begin{array}{l}
\frac{\alpha+3 \beta}{[\alpha+\beta] 3 !} \int_{0}^{1} \frac{t^{2}}{2 !} f(s, u(s)) d s-\frac{\alpha}{[\alpha+\beta] 3 !} \int_{0}^{1} \frac{t^{2}(1-s)^{3}}{2 !} f(s, u(s)) d s- \\
\frac{\beta}{[\alpha+\beta] 2 !} \int_{0}^{1} \frac{t^{2}(1-s)^{2}}{2 !} f(s, u(s)) d s-\int_{0}^{1} \frac{t^{4}}{4 !} f(s, u(s)) d s+\int_{0}^{t} \frac{(t-s)^{4}}{4 !} f(s, u(s)) d s
\end{array}\right| \\
& \leq \frac{\alpha+3 \beta}{[\alpha+\beta] 3 !} \int_{0}^{1} \frac{t^{2}}{2 !}\left(c_{1}|u(s)|+c_{2}\right) d s+\frac{\alpha}{[\alpha+\beta] 3 !} \int_{0}^{1} \frac{t^{2}(1-s)^{3}}{2 !}\left(c_{1}|u(s)|+c_{2}\right) d s \\
& +\frac{\beta}{[\alpha+\beta] 2 !} \int_{0}^{1} \frac{t^{2}(1-s)^{2}}{2 !}\left(c_{1}|u(s)|+c_{2}\right) d s+\int_{0}^{1} \frac{t^{4}}{4 !}\left(c_{1}|u(s)|+c_{2}\right) d s+\int_{0}^{t} \frac{(t-s)^{4}}{4 !}\left(c_{1}|u(s)|+c_{2}\right) d s \\
& \leq \frac{\alpha+3 \beta}{2 ![\alpha+\beta] 3 !}\left(c_{1}|u(s)|\right)+\frac{\alpha}{2 ![\alpha+\beta] 4 !}\left(c_{1}|u(s)|\right)+\frac{\beta}{2 ![\alpha+\beta] 3 !}\left(c_{1}|u(s)|\right)+\frac{1}{4 !}\left(c_{1}|u(s)|\right)+\frac{1}{5 !}\left(c_{1}|u(s)|\right) \\
& \frac{\alpha+3 \beta}{2 ![\alpha+\beta] 3 !}\left(c_{2}\right)+\frac{\alpha}{2 ![\alpha+\beta] 4 !}\left(c_{2}\right)+\frac{\beta}{2 ![\alpha+\beta] 3 !}\left(c_{2}\right)+\frac{1}{4 !}\left(c_{2}\right)+\frac{1}{5 !}\left(c_{2}\right)<\frac{R}{4}+\frac{R}{4}+\frac{R}{4}+\frac{R}{4}=\mathrm{R},
\end{aligned}
$$

which implies that $\|T\| \neq R=\|u\|$, that is a contraction. Then the nonlinear alternative theorem implies that $\mathrm{T}$ has a fixed point $u \in \bar{\Omega}$, that is, problem(3.1),(3.2) and (3.3) has a solution $u \in \bar{\Omega}$.

Finally, we give an example to illustrate the results obtained in this paper.

Example: By using the Equation (3.1) with the conditions (3.2) and (3.3) to solve the boundary value problem

$$
u^{5}(t)=\frac{u+1}{u^{2}+7}
$$

and applying the theorem 3.2 with $\alpha=1$ and $\beta=1$, we found $c_{1}<\min \left(\frac{2 !(\alpha+\beta) 4 !}{5 \alpha+16 \beta}, \frac{5 !}{6}\right)$. So, we conclude that the problem (3.7) has a solution.

\section{References}

[1] R. P. Agarwal and D. Oregan, "Global Existence for Nonlinear Operator Inclusion,” Computers \& Mathematics with Applications, Vol. 38, No. 11-12, 1999, pp. 131 $-139$.

[2] R. P. Agarwal, D. Oregan and P. J. Y. Wong, "Positive 
Solutions of Differential, Difference and Integral Equations,” Kluwer Academic, Dordrecht, 1999.

[3] M. El-Shahed, "Positive Solutions for Nonlinear Singular Third Order Boundary Value Problem," Communications in Nonlinear Science and Numerical Simulation, Vol. 14, No. 2, 2009, pp. 424-429.

[4] D. Guo and V. Lakshmikantham, "Nonlinear Problems in Abstract Cones,” Academic Press, San Diego, 1988.

[5] S. Li, "Positive Solutions of Nonlinear Singular ThirdOrder Two-Point Boundary Value Problem," Journal of Mathematical Analysis and Applications, Vol. 323, No. 1, 2006, pp. 413-425.

[6] H. Sun and W. Wen, "On the Number of Positive Solutions for a Nonlinear Third Order Boundary Value Prob- lem,” International Journal of Difference Equations, Vol. 1, No. 1, 2006, pp. 165-176.

[7] R. P. Agarwal, M. Meehan and D. Oregan, "Fixed Point and Applications," Cambridge University Press, Cambridge, 2001.

[8] B. Yang, "Positive Solutions for a Fourth Order Boundary Value Problem,” Electronic Journal of Qualitative Theory of differential Equations, Vol. 3, No. 1, 2005, pp. 1-17.

[9] S. Q. Zhang, "Existence of Solution for a Boundary Value Problem of Fractional Order," Acta Mathematica Scientica, Vol. 26, No. 2, 2006, pp. 220-228. 